\title{
Apports de lignines et alimentation du lapin en croissance. II. Conséquences sur les performances et la mortalité
}

\author{
JM Perez 1, T Gidenne 1, F Lebas 1, I Caudron 2, \\ P Arveux ${ }^{3}$, A Bourdillon 4, J Duperray ${ }^{5}$, B Messager 6 \\ avec la collaboration technique de $B$ Lamboley ${ }^{1}$, \\ L Mirabito 2, B Perrot 6 , H Rouillère 4, G Troislouches ${ }^{3}$ \\ 1 INRA, station de recherches cunicoles, BP 27, F 31326 Castanet-Tolosan cedex; \\ 2 ITAVI, 28, rue du Rocher, $F 75008$ Paris; \\ 3 UCAAB, BP 19, F 02402 Château-Thierry cedex; \\ 4 Sanders Aliments, BP 32, F 91201 Athis-Mons cedex; \\ 5 Guyomarc'h Nutrition Animale, BP 234, F 56006 Vannes cedex; \\ ${ }^{6}$ Euronutrition, 4, chemin de Villeneuve, Vienne-en-Arthies, F 95510 Vétheuil, France
}

(Reçu le 13 septembre 1993; accepté le 10 janvier 1994)

Résumé - Au total, 2045 lapins en croissance ont été mis en expérience dans 5 stations expérimentales, afin d'étudier les effets respectifs du taux de lignines et de leur nature sur les performances de croissance et la mortalité au cours de la période d'engraissement. Cinq aliments différant essentiellement par le taux $(2,0$ à $7,9 \%)$ et l'origine botanique des lignines ont été distribués à volonté depuis le sevrage (entre 28 et $32 \mathrm{j}$ d'âge selon les stations) jusqu'à l'abattage (entre 66 et $74 \mathrm{j}$ d'âge). Le taux de mortalité $(\mathrm{Tm})$ apparaît étroitement lié $\left(\mathrm{r}^{2}=0,99\right)$ au taux de lignine sulfurique (ADL) selon la relation : $\operatorname{Tm}(\%)=15,8-1,08 \mathrm{ADL}(\%)$; et cela indépendamment de la source de lignines (luzerne déshydratée ou tourteau de pépins de raisins). Parallèlement, l'indice de consommation s'accroît de 0,1 point par point supplémentaire d'ADL dans l'aliment. La lignine sulfurique semble un critère préférable à la lignine permanganate pour prévoir à la fois les performances de croissance et la mortalité des lapereaux.

\section{lapin / alimentation / lignines / croissance / mortalité}

Summary - Dietary lignin in growing rabbits. Il. Consequences on growth performance and mortality. The effects of the level and the origin of the dietary lignin on growth performance and mortality were studied in 5 experimental stations on 2045 growing rabbits. Five diets differing mainly by their acid detergent lignin (ADL) content (2.0-7.9\%) and the origin of the lignin (dehydrated lucerne meal or grape-pip meal) were given ad libitum from weaning (28 and $32 d$ old, depending on the station) to 
slaughter (between 66 and $74 \mathrm{~d}$ old). The mortality rate $(T m)$ was closely related $\left(r^{2}=0.99\right)$ to the $A D L$ level: $T m(\%)=15.8-1.08 \mathrm{ADL}(\%)$; without influence of the botanical origin of the lignin. The feed conversion ratio increased by 0.1 points per additional point of dietary $A D L$. $A D L$ was preferred to permanganate lignin for the prediction of growth performances and mortality in the rabbit.

rabbit / feeding / lignin / growth / mortality

\section{INTRODUCTION}

Un apport alimentaire minimum de parois végétales est indispensable au lapin pour assurer un fonctionnement digestif normal sans perturbation du transit (Laplace, 1978) et éviter ainsi l'apparition d'entérites souvent mortelles (Peeters et Charlier, 1984). $\mathrm{Ce}$ besoin spécifique en fibres limite les possibilités d'accroissement de la concentration énergétique des régimes et par voie de conséquence leur efficacité alimentaire. Cet antagonisme entre performances de croissance et bon état sanitaire constitue un problème majeur en nutrition cunicole. Sa résolution passe par une définition précise des apports en constituants pariétaux. Or, le besoin en fibres du lapin demeure encore très mal identifié à la fois quantitativement et qualitativement. Actuellement, il repose essentiellement sur le critère cellulose brute, dont la signification biochimique et nutritionnelle a été maintes fois mise en cause (Van Soest et McQueen, 1973; Sauvant, 1981; Perez, 1991). Ainsi, il n'est pas surprenant, malgré le nombre important de travaux déja réalisés, qu'aucune relation claire n'ait pu être établie entre le taux de cellulose Weende et la mortalité en engraissement (Heckman et Mehner, 1970 ; Colin et al, 1976 ; Franck et Coulmin, 1978 ; Licois et al,1980; De Blas et al, 1986 ; Champe et Maurice, 1983). De la même façon, plusieurs expérimentations basées sur des apports variables en certaines fractions pariétales selon Van Soest (lignocellulose et hémicelluloses) demeurent encore insuffisantes pour dégager des conclusions fiables (Lebas et al, 1989 ; Maitre et al, 1990).
Cette étude a pour but d'évaluer l'intérêt de prendre en compte un indicateur des lignines, pour prévoir les performances de croissance et prévenir les pertes de lapereaux en engraissement. La teneur en lignines a été appréciée par 2 méthodes analytiques à partir du résidu ADF (acid detergent fiber) selon Van Soest : I'isolement d'un résidu ADL (acid detergent lignin) après hydrolyse dans l'acide sulfurique concentré ou l'oxydation par le permanganate. Ce travail fait suite à l'étude traitant de l'incidence des lignines sur la digestion et le transit (Gidenne et Perez, 1994). II s'agit cette fois de conduire des expérimentations sur des effectifs importants d'animaux et dans des conditions variées d'élevage, afin d'obtenir des résultats transposables à des élevages de production. Précisons que les fortes variabilités observées classiquement chez le lapin pour la mortalité et les performances de croissance nécessitent la mise en place de tels dispositifs expérimentaux (Morin et al, 1979).

\section{MATÉRIEL ET MÉTHODES}

\section{Animaux et conditions expérimentales}

Le même protocole a été appliqué dans 5 stations expérimentales cunicoles, respectivement dans les installations de I'ITAVI (Rambouillet) et de firmes d'alimentation du bétail : la CCPA (Vienne-en-Arthies), Guyomarc'h (Saint-Nolff), Sanders (Sourches) et l'UCAAB (Montfaucon). Les essais réalisés dans ces stations diffèrent par les effectifs mis en place, les types génétiques et le mode de logement des animaux. Les 
conditions expérimentales sont résumées dans le tableau I. Au total, 2045 lapereaux ont été mis en expérimentation à raison de 409 animaux par régime.

\section{Aliments expérimentaux}

Cinq aliments, différant essentiellement par le taux et la nature des lignines, ont été comparés : un aliment de référence ( $\mathrm{R} 0$ ) à faible teneur en lignine $A D L$, et des aliments à taux croissants d'ADL provenant pour un tiers, soit de luzerne déshydratée (régimes $L 1$ et $L 2$ ), soit de tourteau de pépins de raisin (régimes $\mathrm{P} 1$ et $\mathrm{P} 2$ ). Leur composition centésimale et leurs caractéristiques analytiques ont été rapportées précédemment (Gidenne et Perez, 1994). Les aliments (granulés de $4 \mathrm{~mm}$ de diamètre) ont été fabriqués en une seule fois (ITCF, Boigneville) à partir des mêmes lots de matières premières. Ils ont été distribués à volonté depuis le sevrage (entre 28 et 32 j d'âge selon les sites) jusqu'à l'abattage (entre 66 et 74 j d'âge).

\section{Mesures sur animaux}

Les lapereaux ont été pesés individuellement au sevrage, à 49 j d'âge et au stade final d'abattage. Les consommations d'aliment ont été mesurées par période et par cage. La mortalité a été contrô-

Tableau I. Modalités expérimentales dans les différents sites

$$
\begin{gathered}
\text { Site Effectif Effectif Type Âge Âge } \\
\text { par par génétique début d'abattage } \\
\text { régime cage }
\end{gathered}
$$

\begin{tabular}{rrrlll}
\hline 1 & 72 & 6 & Hycole & 32 & 74 \\
2 & 63 & 7 & Hyplus & 28 & 66 \\
3 & 114 & 6 & Hyplus & 28 & 70 \\
4 & 120 & 6 & Hyplus & 28 & 71 \\
5 & 40 & 5 & CAx NZa & 32 & 73
\end{tabular}

Total 409

a Croisement Californien x Néo-Zélandais Blanc. lée quotidiennement. En cas de mortalité, l'aliment restant dans la trémie était pesé afin d'estimer la consommation réelle des animaux sur la base de la durée de présence et de l'effectif des lapereaux par cage.

\section{Analyse statistique}

Les résultats expérimentaux ont d'abord été analysés par site expérimental, en utilisant notamment les techniques d'analyses de variance et de covariance selon la procédure GLM du logiciel SAS (1988). Cette analyse a été suivie par un traitement d'ensemble des données des 5 essais en prenant en compte outre les effets principaux "régime" et "site", l'effet "répétition intra-site (bande)" et les interactions "site $x$ régime". Les comparaisons multiples de moyennes ont été réalisées à l'aide du test de Bonferroni. Les résultats de mortalité ont été analysés selon la méthode de $\mathrm{K}$ Pearson (distribution du $\chi^{2}$ ), complétée par la procédure proposée par Mantel et Haenszel (Mantel, 1963) pour tester la linéarité de réponse du taux de mortalité en fonction du taux de lignine.

\section{RÉSULTATS}

\section{Performances de croissance}

Au cours de la phase initiale de croissance (du sevrage à 49 j d'âge), aucune interaction site $x$ régime n'a été mise en évidence quel que soit le critère considéré (tableau II). Les poids moyens de départ varient selon les sites d'expérience entre 605 et $843 \mathrm{~g}$ (fig 1) en relation avec les âges au sevrage pratiqués (28 ou 32 j) et les types génétiques utilisés. Mais la prise en compte du poids au sevrage comme covariable n'a pas permis d'améliorer la précision de l'analyse. Durant cette période, les gains de poids diffèrent significativement selon les régimes avec un écart maximum de $4 \mathrm{~g}$ par jour (soit $10 \%$ environ en valeur relative) entre les aliments $\mathrm{R} 0$ et $\mathrm{P} 2$ à teneurs extrêmes en lignine ADL. L'ampleur des variations de 
Tableau II. Performances moyennes au cours de la période de croissance (du sevrage à 49 j d'âge).

\begin{tabular}{|c|c|c|c|c|c|c|c|c|c|}
\hline Régimes & L2 & $\angle 1$ & RO & $P 1$ & $P 2$ & $E T R^{1}$ & & ification & statistique? 2 \\
\hline Lignine $A D L \%$ & 5,9 & 4,0 & 2,0 & 4,7 & 7,6 & & Site & Régime & Interaction \\
\hline Poids au sevrage (g) & 674 & 672 & 672 & 677 & 673 & 15 & $\star \star$ & NS & NS \\
\hline Gain de poids $(g / j)$ & $46,0^{b}$ & $46,4^{a b}$ & $43,8^{c}$ & $45,4^{b c}$ & $48,0^{a}$ & 3,9 & ** & $\star \star$ & NS \\
\hline $\begin{array}{l}\text { Consommation } \\
\text { (g/j) } \\
\text { (MJ ED/j) }\end{array}$ & $\begin{array}{c}101,9^{\mathrm{b}} \\
1,04^{\mathrm{a}}\end{array}$ & $\begin{array}{c}96,6^{\mathrm{c}} \\
1,03^{\mathrm{a}}\end{array}$ & $\begin{array}{c}85,2^{\mathrm{d}} \\
0,94^{\mathrm{b}}\end{array}$ & $\begin{array}{c}96,8^{\mathrm{c}} \\
0,97^{\mathrm{b}}\end{array}$ & $\begin{array}{r}109,1^{\mathrm{a}} \\
1,01^{\mathrm{a}}\end{array}$ & $\begin{array}{l}7,3 \\
0,07\end{array}$ & $\stackrel{\star *}{* *}$ & $\begin{array}{l}\star \star \\
\star *\end{array}$ & $\begin{array}{l}\text { NS } \\
\text { NS }\end{array}$ \\
\hline $\begin{array}{l}\text { Indice de consommati } \\
\text { (kg/kg gain) } \\
\text { (MJ ED/kg gain) }\end{array}$ & $\begin{array}{c}n, 26^{a} \\
23,1^{a}\end{array}$ & $\begin{array}{c}2,12^{\mathrm{b}} \\
22,6^{\mathrm{a}}\end{array}$ & $\begin{array}{c}1,97^{\mathrm{c}} \\
21,8^{\mathrm{b}}\end{array}$ & $\begin{array}{c}2,16^{b} \\
21,7^{b}\end{array}$ & $\begin{array}{c}2,31^{\mathrm{a}} \\
21,5^{\mathrm{b}}\end{array}$ & $\begin{array}{l}0,16 \\
1,6\end{array}$ & $* *$ & $\stackrel{* *}{* *}$ & $\begin{array}{l}\text { NS } \\
\text { NS }\end{array}$ \\
\hline
\end{tabular}

1 ETR : écart type résiduel. ${ }^{2}$ NS : $P>0,10 ;{ }^{*} P<0,05 ;{ }^{* *}: P<0,01$, a,b : les moyennes ayant une lettre en commun ne differrent pas au seuil $P=0,05$.

consommation d'aliment (+28\%) en faveur des animaux recevant les régimes à fort taux d'ADL est à mettre en relation avec les teneurs en énergie digestible (ED) des régimes mesurées lors de la première partie de ce travail (Gidenne et Perez, 1994). De fait, exprimée sur la base de l'ED, la consommation est moins affectée par le régime alimentaire. Cependant, il subsiste des écarts significatifs entre lots pour l'ingestion d'ED au bénéfice surtout des régimes $L 1$ et $L 2$ (+ $10 \%$ par rapport au régime $R 0)$. Pour ces derniers, la consommation supérieure d'ED ne se traduit pas par des variations de même intensité de la vitesse de croissance. II en résulte une détérioration significative de leur efficacité énergétique comparativement à l'aliment de référence RO (22,9 vs 21,8 MJ d'ED par kg de gain). En revanche, l'accroissement de l'ingestion d'énergie pour les régimes de type $P$ est de même amplitude que celui du gain de poids, de sorte que les indices de consommation énergétique sont identiques à celui du lot Ro.

Au cours de la période de finition (tableau III), les gains de poids ne diffèrent pas en moyenne d'un traitement à l'autre, contrairement à la période initiale de croissance. Il faut noter néanmoins une interaction site $x$ régime pour ce critère, qui s'explique essentiellement par de plus faibles valeurs du croît journalier dans un site avec les animaux du lot $\mathrm{P} 2$, contrairement à la tendance générale. Comme précédemment, la consommation d'aliment s'accroît avec le taux d'ADL $\left(r^{2}=0,94\right)$ mais les écarts entre lots extrêmes sont plus faibles ( $+18 \%$ entre P2 et $R 0)$. Les vitesses de croissance étant similaires selon les traitements, l'indice de consommation suit l'évolution de l'ingestion. En revanche, la consommation d'énergie digestible, et corrélativement les indices de consommation énergétique sont significativement plus élevés pour les régimes de type $L$ comme au cours de la première période.

\section{Mortalité}

Les résultats moyens de mortalité sont présentés tous sites confondus dans le 
Tableau III. Performances moyennes au cours de la période de finition (de 49 j d'âge à l'abattage).

\begin{tabular}{|c|c|c|c|c|c|c|c|c|c|}
\hline $\begin{array}{l}\text { Régimes } \\
\text { Lignine } A D L(\%)\end{array}$ & $\begin{array}{l}L 2 \\
5,9\end{array}$ & $\begin{array}{l}L 1 \\
4,0\end{array}$ & $\begin{array}{l}R 0 \\
2,0\end{array}$ & $\begin{array}{r}P 1 \\
4,7\end{array}$ & $\begin{array}{l}P 2 \\
7,6\end{array}$ & ETR 1 & Site & $\begin{array}{r}\text { Signifi } \\
\text { statist } \\
\text { Régime }\end{array}$ & $\begin{array}{l}\text { ation } \\
\text { que }{ }^{2} \\
\text { Interaction }\end{array}$ \\
\hline Gain de poids $(g / j)$ & 35,5 & 36,4 & 36,7 & 37,1 & 35,8 & 3,6 & ** & NS & ** \\
\hline $\begin{array}{l}\text { Consommation } \\
(\mathrm{g} / \mathrm{j}) \\
(\mathrm{MJ} \text { ED/j) }\end{array}$ & $\begin{array}{r}151,8^{a} \\
1,55^{a}\end{array}$ & $\begin{array}{r}144,9^{\mathrm{b}} \\
1,55^{\mathrm{a}}\end{array}$ & $\begin{array}{r}131,2^{c} \\
1,45^{b}\end{array}$ & $\begin{array}{r}145,4^{b} \\
1,46^{b}\end{array}$ & $\begin{array}{r}155,4^{a} \\
1,44^{b}\end{array}$ & $\begin{array}{c}10,3 \\
0,11\end{array}$ & ** & 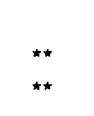 & $\begin{array}{l}\text { NS } \\
\text { NS }\end{array}$ \\
\hline $\begin{array}{l}\text { Indice de consomm } \\
\text { (kg/kg gain) } \\
\text { (MJ ED/kg gain) }\end{array}$ & $\begin{array}{l}4,32^{a} \\
44,1^{a}\end{array}$ & $\begin{array}{l}4,03^{b} \\
43,1^{a}\end{array}$ & $\begin{array}{c}3,62^{c} \\
39,9^{b}\end{array}$ & $\begin{array}{l}4,01^{b} \\
40,3^{b}\end{array}$ & $\begin{array}{r}4,35^{a} \\
40,4^{b}\end{array}$ & $\begin{array}{l}0,49 \\
5,0\end{array}$ & $\star *$ & ** & $\begin{array}{l}\text { NS } \\
\text { NS }\end{array}$ \\
\hline
\end{tabular}

1,2 Voir tableau II.

Tableau IV. Mortalité des lapereaux en engraissement.

\begin{tabular}{|c|c|c|c|c|c|c|c|c|c|}
\hline \multirow{2}{*}{$\begin{array}{l}\text { Régimes } \\
\text { Lignine ADL (\%) } \\
n\end{array}$} & \multirow{2}{*}{$\begin{array}{l}L 2 \\
5,9 \\
409\end{array}$} & \multirow{2}{*}{$\begin{array}{r}L 1 \\
4,0 \\
409\end{array}$} & \multirow{2}{*}{$\begin{array}{r}R O \\
2,0 \\
409\end{array}$} & \multirow{2}{*}{$\begin{array}{r}P 1 \\
4,7 \\
409\end{array}$} & \multirow{2}{*}{$\begin{array}{r}P 2 \\
7,6 \\
409\end{array}$} & \multicolumn{2}{|c|}{$\begin{array}{c}\text { Effet } \\
\text { Régime? }\end{array}$} & \multicolumn{2}{|c|}{$\begin{array}{c}\text { Effet } \\
\text { Taux } A D L^{3}\end{array}$} \\
\hline & & & & & & $\chi^{2}$ & Prob & $Q_{M H}$ & Prob \\
\hline \multicolumn{10}{|l|}{ Période sevrage - $49 j$} \\
\hline $\begin{array}{l}\text { Nombre de morts } \\
\text { Taux de mortalité }(\%)^{1}\end{array}$ & $\begin{array}{l}20 \\
4,89\end{array}$ & $\begin{array}{l}18 \\
4,40\end{array}$ & $\begin{array}{l}33 \\
8,07\end{array}$ & $\begin{array}{l}18 \\
4,40\end{array}$ & $\begin{array}{l}13 \\
3,18\end{array}$ & 11,6 & 0,02 & 7,40 & 0,006 \\
\hline \multicolumn{10}{|l|}{ Période $49 j$ - abattage } \\
\hline $\begin{array}{l}\text { Nombre de morts } \\
\text { Taux de mortalité (\%) } 1\end{array}$ & $\begin{array}{l}18 \\
4,40\end{array}$ & $\begin{array}{l}28 \\
6,85\end{array}$ & $\stackrel{24}{5,87}$ & $\begin{array}{l}26 \\
6,36\end{array}$ & $\begin{array}{l}19 \\
4,65\end{array}$ & 3,7 & 0,45 & 2,3 & 0,13 \\
\hline \multicolumn{10}{|l|}{ Période totale } \\
\hline $\begin{array}{l}\text { Nombre de morts } \\
\text { Taux de mortalité (\%) } 1\end{array}$ & $\begin{array}{l}38 \\
9,29\end{array}$ & $\begin{array}{l}46 \\
11,25\end{array}$ & $\begin{array}{l}57 \\
13,94\end{array}$ & $\begin{array}{l}44 \\
10,76\end{array}$ & $\begin{array}{l}32 \\
7,82\end{array}$ & 9,1 & 0,06 & 8,7 & 0,003 \\
\hline
\end{tabular}

1 Taux de mortalité exprimé en pourcentage de l'effectif initial de lapereaux par régime $(n=409) .2$ Test de $\chi^{2}$ selon K Pearson. ${ }^{3}$ Test de $\chi^{2}$ selon Mantel-Haenszel (Mantel, 1963).

tableau IV. Dans la majorité des cas, la mort est associée à une diarrhée ; les morts accidentelles n'ont pas été comptabilisées ici. On observe globalement un effet significatif (valeur du $\chi^{2}=9,1 ; P=0,06$ ) du régime alimentaire sur les pertes de lapereaux au cours de la période totale d'engraissement, les taux de mortalité s'échelonnant entre $7,8 \%$ (régime $\mathrm{P} 2$ ) et $14 \%$ (régime $\mathrm{R} 0$ ). Cet effet global résulte essentiellement d'une influence très forte du régime alimentaire sur la mortalité au cours de la période initiale 
de croissance (valeur du $\chi^{2}=11,6$; $P=0,02$ ). De fait, entre le sevrage et $49 \mathrm{j}$ d'âge, les pertes d'animaux sont multipliées par 2 avec le régime $\mathrm{RO}$ comparativement aux autres régimes mieux pourvus en lignine. Au cours de la période de finition, l'effet du régime n'est plus significatif en raison d'une mortalité importante pour les régimes à teneurs modérées en lignines (L1 et $\mathrm{P} 1$ ). Il en résulte une relation linéaire très étroite entre la teneur en ADL et la mortalité durant la totalité de la période expérimentale (test de Mantel-Haenszel significatif au seuil $P=0,003)$. Les différences observées entre les taux élevés et intermédiaires d'ADL $(\mathrm{L} 2+\mathrm{P} 2$ vs $\mathrm{L} 1+\mathrm{P} 1)$ demeurent significatives $(P=0,09)$, alors que la nature des lignines (comparaison des régimes $\mathrm{P}$ et $\mathrm{L}$ ) est sans influence sur la mortalité.

Indépendamment du régime alimentaire, le taux de mortalité varie considérablement selon le site expérimental (entre 3,5 et $17 \%$ en moyenne). Cet effet «site» est sans relation avec l'âge au sevrage ou le poids initial des lapereaux (fig 1).

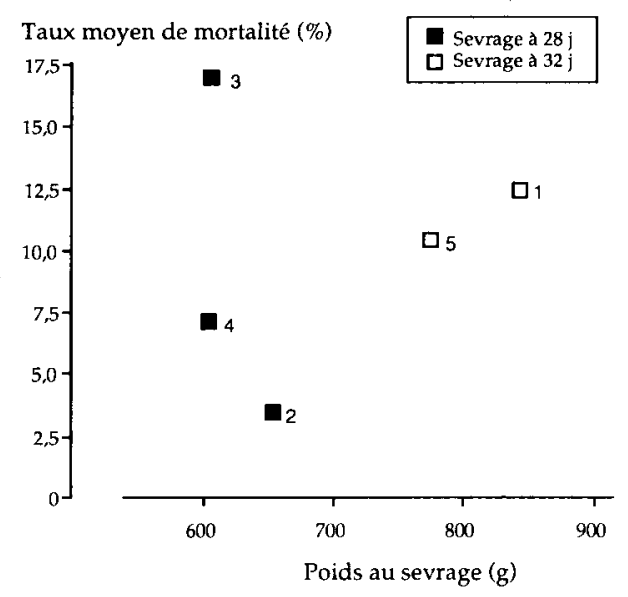

Fig 1. Taux moyen de mortalité (\%) selon le site, l'âge et le poids au sevrage. $1,2 \ldots: n^{\circ}$ du site expérimental.

\section{DISCUSSION}

Sur le plan méthodologique, l'intérêt de cette étude réside dans la puissance expérimentale du dispositif (409 animaux par régime) et la diversité des conditions d'élevage grâce à la mise en commun de moyens d'étude, ainsi que dans l'application d'un protocole standard de contrôle des performances de croissance. Soulignons le niveau satisfaisant de ces dernières : en moyenne plus de $40 \mathrm{~g}$ de croît quotidien pour une consommation journalière d'aliment de $123 \mathrm{~g}$, soit un indice de consommation d'environ $3 \mathrm{~kg}$ par $\mathrm{kg}$ de gain.

On admet généralement que le lapin en croissance, recevant des aliments équilibrés, est capable d'ajuster son ingestion alimentaire en réponse à des variations de concentration énergétique de manière à consommer quotidiennement la même quantité d'énergie digestible (ED) et de maintenir ainsi sa vitesse de croissance (Lebas, 1975 ; Bombeke et al, 1978 ; Spreadbury et Davidson, 1978 ; Lebas et al, 1982 ; Partridge et al, 1989). Cette régulation est possible dans une plage de concentration comprise entre 9,2 et 13,4 MJ d'ED par kg d'aliment brut. Dans la gamme de concentration des teneurs en ED mesurées sur les aliments expérimentaux, soit 9,3 à $11 \mathrm{MJ}$ par kg (Gidenne et Perez, 1994), la régulation de l'ingestion devrait jouer pleinement. Or, si la teneur en ED reste ici un facteur explicatif important de la consommation d'aliment, la liaison entre ces 2 variables n'est pas très forte $\left(r^{2}=0,78\right.$ sur la période totale) et la consommation d'ED demeure significativement supérieure pour les régimes de type $L$ sur l'ensemble des périodes, bien que les écarts entre lots restent modestes $(+7 \%)$. La capacité d'ingestion des lapereaux, exprimée en ED par $\mathrm{kg}$ de poids métabolique (poids vif à $49 \mathrm{j}$ à la puissance 0,75 ) s'élève à $0,95 \mathrm{MJ}$ par jour pour les régimes de type $L$ contre $0,89 \mathrm{MJ}$ pour les autres régimes sur la tota- 
lité de la période d'engraissement. Remarquons que, dans la gamme très large des teneurs en ADL testées, la corrélation entre la consommation brute d'aliment et le taux d'ADL est très forte $\left(r^{2}=0,98\right.$ sur la période totale), mais demeure imprécise dans la zone usuelle de concentration (entre 4 et $5 \%$ ).

En ce qui concerne la vitesse de croissance, les différences entre régimes, bien que significatives, demeurent faibles si l'on se réfère à l'ensemble de la période d'engraissement (de l'ordre de $1 \mathrm{~g}$ par jour pour un gain moyen de $40,4 \mathrm{~g} / \mathrm{j}$ ). Les seuls écarts notables ont trait à la période initiale de croissance où les gains de poids atteignent des valeurs élevées ( $46 \mathrm{~g}$ en moyenne par jour). Cette période initiale de forte expression des potentialités de croissance des animaux correspond également à une phase de sensibilité accrue des lapereaux aux conditions d'alimentation et de milieu. On ne peut donc exclure que ces écarts de croissance au bénéfice des régimes riches en lignines soient la conséquence d'un meilleur équilibre physiopathologique des animaux recevant ces régimes dès le sevrage.

Les variations d'indice de consommation (IC) entre lots sont identiques quelle que soit la période. Si l'on considère la période totale, il ressort une relation très étroite entre la teneur en ADL et I'IC, indépendamment de la nature des lignines (régimes $L$ ou $P$ ), qui s'exprime de la manière suivante:

$$
\text { IC }(\mathrm{kg} / \mathrm{kg} \text { gain })=2,58+0,10 \mathrm{ADL}(\%)
$$

$\mathrm{r}^{2}=0,94, \quad$ ETR $=0,06, \quad \mathrm{CV}=1,9 \%$

Cette relation indique un accroissement de 0,1 point d'indice de consommation, soit une dépense supplémentaire de $100 \mathrm{~g}$ d'aliment par kg de gain, par point d'augmentation de lignine sulfurique dans l'aliment. Dans nos conditions expérimentales, ce dernier critère apparaît davantage explicatif que l'ED $\left(r^{2}=0,71 ; C V=4,2 \%\right)$ ou que la lignine per- manganate $\left(r^{2}=0,89 \mathrm{CV}=2,6 \%\right)$ pour prédire l'efficacité alimentaire des régimes.

Dans le même sens, l'indice de consommation exprimé sur la base de l'énergie digestible n'est pas identique quel que soit le régime. De fait, s'il ne varie pas avec la teneur en lignines entre l'aliment de référence $(R 0)$ et les aliments de type $P$, en revanche il se détériore nettement quand on passe de R0 à L2 (+2,5 MJ ED/kg gain sur la période totale). Sachant que les apports azotés sont équivalents selon les régimes (Gidenne et Perez, 1994), une des explications pourrait résider dans les proportions accrues de pulpes de betteraves dans les régimes $\mathrm{R} 0, \mathrm{P} 1$ et $\mathrm{P} 2$, qui sont susceptibles d'accroître les poids du tube et des contenus digestifs pour un même poids d'abattage (Candau et al, 1978 ; Pérez De Ayala, 1989 ; Garcia et al, 1993).

La mortalité des lapereaux en engraissement constitue une préoccupation majeure des éleveurs en raison de ses répercussions économiques évidentes. Évaluées à $12 \%$ en moyenne d'après les résultats de la Gestion technico-économique (Ponsot, 1993), ces pertes sont essentiellement d'origine digestive (diarrhées). Mais les relations entre l'alimentation et la pathologie digestive demeurent encore peu élucidées (Peeters, 1988). Cette étude avait pour objectif principal de tenter de relier la mortalité à un critère pariétal, en l'occurrence les fractions lignines appréciées par 2 méthodes : lignine sulfurique (ADL) ou lignine au permanganate.

D'après nos résultats, obtenus sur un effectif initial dépassant 2000 lapereaux, il apparaît une relation très étroite entre la teneur en $\mathrm{ADL}$ des régimes et le taux de mortalité (Tm) sur la période totale d'engraissement :

$$
\operatorname{Tm}(\%)=15,8-1,08 \mathrm{ADL}(\%)
$$

$r^{2}=0,99 \quad$ ETR $=0,27 \quad C V=2,6 \%$ 
Cette relation indique une baisse de 1 point de la mortalité par point d'augmentation du taux d'ADL de l'aliment (fig 2). À notre connaissance, c'est la première fois qu'une liaison aussi forte est enregistrée entre un critère nutritionnel et la mortalité des lapereaux. Jusqu'à présent, les études conduites sur ce thème ont surtout tenté de relier l'importance des pertes avec la teneur en cellulose brute des régimes. Elles ont débouché le plus souvent sur des résultats décevants même pour des plages très larges d'apport. Ainsi, par exemple, aucune relation entre ces 2 critères n'a pu être mise en évidence pour un ensemble de régimes titrant entre 12 et $16 \%$ de cellulose brute (Lebas, 1989). La raison est probablement à rechercher d'abord dans la signification même du résidu organique de Weende, dont les limites ont été maintes fois soulignées, surtout pour les mélanges alimentaires. Estimateur incomplet et non représentatif des parois végétales, la cellulose brute est en effet un mauvais descripteur pariétal pour les régimes complexes incluant des sources de fibres appartenant à des familles végétales très diversifiées, comme

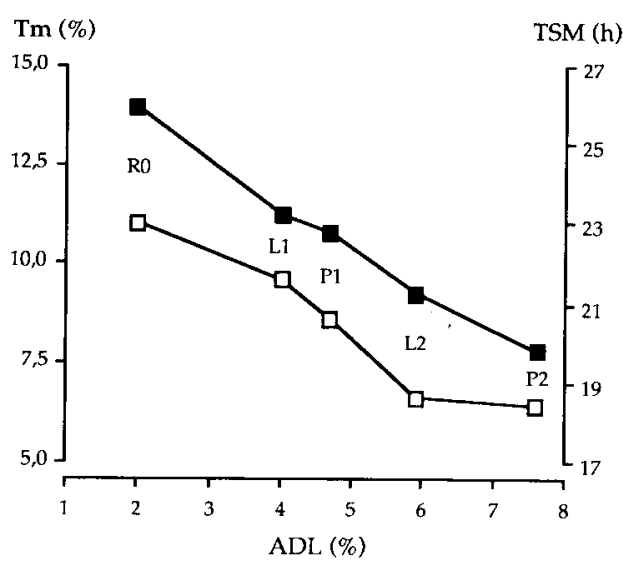

Fig 2. Influence du taux de lignine (ADL) sur le transit et la mortalité. $\square$ Tm : taux moyen de mortalité entre le sevrage et l'abattage ; $\square$ TSM : temps de séjour moyen total des aliments. cela est de pratique courante en nutrition cunicole. En revanche, les résidus lignines seraient mieux à même de refléter cette diversité tout en constituant des indicateurs d'indigestibilité des aliments. S'agissant des estimateurs des teneurs en lignines, la lignine sulfurique semble préférable à la lignine au permanganate $\left.(\mathrm{Li} \mathrm{KMnO})_{4}\right)$. Avec ce dernier critère, la liaison avec la mortalité est en effet moins étroite :

$$
\operatorname{Tm}(\%)=16,8-1,47 \mathrm{Li} \mathrm{KMnO}_{4}(\%)
$$

$r^{2}=0,78 \quad E T R=1,24 \quad C V=11,7 \%$

Rappelons toutefois que ce dernier critère n'a été analysé que dans un seul laboratoire contre 5 sites de dosage pour la lignine sulfurique.

Ajoutons qu'il convient d'être prudent dans l'interprétation des résultats quand on raisonne en termes de pourcentage de tel ou tel constituant du régime en relation avec la mortalité par exemple. Les lapins étant alimentés à volonté, il faut prendre en compte les quantités de nutriments effectivement ingérées. Ainsi, on observe une forte corrélation entre la teneur en amidon des régimes et les pertes de lapereaux $\left(r^{2}=0,97\right)$, alors que la corrélation entre les quantités d'amidon ingérées et la mortalité est quasi nulle $\left(r^{2}=0,03\right)$. En revanche, la corrélation entre les quantités de lignine sulfurique (ADL) ingérées et le taux de mortalité demeure hautement significative $\left(r^{2}=0,99\right)$.

Par ailleurs, contrairement à une notion généralement admise, nous n'avons pas observé dans cette étude une mortalité moyenne plus forte en période initiale de croissance $(4,9 \%)$ qu'en période de finition $(5,9 \%)$. Dans nos conditions expérimentales, nous n'avons pas non plus enregistré de troubles digestifs tels que des parésies cæcales malgré des ratios MAD/ED élevés (notamment avec le régime $\mathrm{P} 2$ ), comme l'ont noté d'autres expérimentateurs (De Blas et al, 1981). 
En définitive, l'intérêt majeur de cette double étude est d'améliorer les connaissances relatives aux interactions nutrition et pathologie digestive chez le lapin en croissance en faisant appel à des techniques complémentaires (bilans digestifs, mesures de transit et de mortalité). Ainsi, pour la première fois nous avons pu observer simultanément une relation entre la réduction des apports de fibres lignifiées, le ralentissement du transit digestif et la mortalité en engraissement (fig 2). Cela corrobore les hypothèses généralement avancées (Cheeke, 1987 ; Lebas, 1989), mais rarement étayées par des résultats expérimentaux.

\section{CONCLUSION}

Les résultats de cette étude font ressortir un effet favorable du taux de lignine sur la mortalité du lapin en croissance et cela indépendamment de la nature des lignines. En revanche, les performances d'engraissement sont influencées par l'origine botanique des lignines. D'une façon générale, la lignine ADL semble préférable à la lignine permanganate pour prévoir la valeur nutritive des aliments (digestibilité, transit), les performances de croissance et la mortalité.

\section{REMERCIEMENTS}

La présente étude a été réalisée sous la responsabilité de I'INRA avec le soutien financier du Fonds SYPRAM. Le SYPRAM a été créé en 1990 avec des fonds apportés par les adhérents de l'AMEB (Association pour le maintien de l'élevage en Bretagne), du SNIA (Syndicat national des industries de la nutrition animale) et du SYNCOPAC (Fédération nationale des coopératives de production et d'alimentation animales) en vue de faciliter toute action d'intérêt collectif au profit des entreprises du secteur de l'alimentation animale.

\section{RÉFÉRENCES}

Bombeke A, Okerman F, Moermans R (1978) Influence de la granulation à sec et à la vapeur des rations à teneur différente en énergie sur les résultats de production des lapins de chair. Rev Agric 361, 945-955

Champe KA, Maurice DW (1983) Response of early weaned rabbits to source and level of dietary fiber. $\checkmark$ Anim Sci 56, 1105-1114

Cheeke PR (1987) Nutrition-disease interrelationships. In : Rabbit Feeding and Nutrition, Academic Press Inc, 176-200

Candau M, Delpon G, Fioramonti J (1978) Influence de la nature des glucides membranaires sur le développement anatomo-fonctionnel du tractus digestif

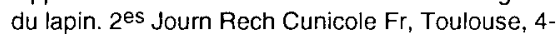
5 avril 1978, Communication $n^{\circ} 1,1.1-1.4$, ITAVI Ed, Paris

Colin M, Maire C, Vaissaire J, Renault L (1976) Étude expérimentale du remplacement dans les aliments pour lapins de la cellulose par des lests minéraux: sable et vermiculite. Recl Méd Vét 152, 457-465

De Blas JC, Pérez E, Fraga MJ, Rodrigez JM, Galvez JF (1981) Effect of diet on feed intake and growth of rabbits from weaning to slaughter at different ages and weigh. J Anim Sci 52, 1225-1232

De Blas JC, Santoma G, Carabano R, Fraga MJ (1986) Fiber and starch levels in fattening rabbit diets. $J$ Anim Sci 63, 1897-1904

Franck Y, Coulmin JP (1978) Utilisation de la paille de blé broyée comme source de cellulose dans les aliments lapins à l'engraissement ; comparaison de deux taux de cellulose. 2es Journ Rech Cunicole Fr, Toulouse, 4-5 avril 1978. Communication n 10, 10.1-10.6, ITAVI Ed, Paris

Garcia G, Galvez JF, De Blas JC (1993) Effect of substitution of sugar beet pulp for barley in diets for finishing rabbits on growth performance and on energy and nitrogen. J Anim Sci 71, 1823-1830

Gidenne T, Perez JM (1994) Apports de lignines et alimentation du lapin en croissance. I. Conséquences sur la digestion et le transit. Ann Zootech 43, 313-322

Heckmann FW, Mehner A (1970) Versuche über den Eiweiss und Rohfasergehalt in Alleinfutter für Jungmastkaninchen. Arch Geflügelzucht Kleintierkd 19,2943

Laplace JP (1978) Le transit digestif chez les monogastriques. III. Comportement (prise de nourriture-cæcotrophie), motricité et transit digestif et pathogénie des diarrhées chez le lapin. Ann Zootech 27, 225-265

Lebas $F$ (1975) Influence de la teneur en énergie de l'aliment sur les pertormances de croissance chez le lapin. Ann Zootech 24, 281-288

Lebas F (1989) Besoins nutritionnels des lapins. Revue bibliographique et perspectives. Cuni-Sciences 5 (2), 1-28 
Lebas F, Laplace JP, Droumenq (1982) Effets de la teneur en énergie de l'aliment chez le lapin. Variations en fonction de l'âge des animaux et de la séquence des régimes alimentaires. Ann Zootech $31,233-256$

Lebas F, Maitre I, Arveux P, Bouillet A, Bourdillon A, Duperray J, Saint-Cast $Y$ (1989) Taux d'hémicellulose et performances de croissance du lapin de chair. L'Éleveur de Lapins (27), 40-43

Licois D, Coudert P, Colin M (1980) Essai d'induction de la diarrhée chez le lapereau à l'aide d'aliments comportant différentes teneurs en cellulose. Ann Rech Vét 11, 279-284

Maitre I, Lebas F, Arveux P, Bourdillon A, Duperray J, Saint-Cast $Y$ (1990) Taux de lignocellulose (ADF de Van Soest) et performances de croissance du lapin de chair. 5es Journ Rech Cunicole Fr, Paris, 12-13 décembre 1990, Communication $n^{\circ} 56,56.1-56.12$ ITAVI Ed, Paris

Mantel N (1963) Chi-square tests with one degree of freedom: extensions of the Mantel-Haenszel procedure. J Am Stat Assoc 58, 690-700

Morin P, Seroux M, Piganeau P (1979) Méthodes d'expérimentation sur le lapin. Perspectives Agricoles (23), 64-72

Partridge GG, Garthwaite PH, Findlay M (1989) Protein and energy retention by growing rabbits offered diets with increasing proportions of fibre. J Agric Sci UK $112,171-178$
Peeters JE (1988) Recent advances in intestinal pathology of rabbits and further perspectives. Proc 4th Congress of the World Rabbit Science Association, Budapest, Hungary, 10-14 October 1988, WRSA Ed Vol 2, 293-313.

Peeters JE, Charlier GJ (1984) Le complexe entérite du lapin de chair en élevage rationnel. Cuni-Sciences 2 (3), 13-26

Perez JM (1991) Intérêt et limites des modèles de prévision de la valeur énergétique des aliments destinés au porc. Thèse de doctorat, université de Montpellier II, $196 \mathrm{p}$

Pérez de Ayala PM (1989) Utilizacion de distintos tipos de fibra por los conejos en cebo. Tesis doctoral, Univ Politec Madrid, ETS Ing Agronom, $115 p$

Ponsot JF (1993) Gestion technico-économique : bilan et résultats 1992. Cuniculture 20, 185-192

SAS (1988) SAS/STAT Guide for Personal Computers (Release 6.03). SAS Inst Inc, Cary, NC, $1028 \mathrm{p}$

Sauvant $D$ (1981) Prévision de la valeur énergétique des aliments concentrés et composés pour les ruminants. In: Prévision de la valeur nutritive des aliments des ruminants (C Demarquilly, ed), INRA, Paris, 237-258

Spreadbury D, Davidson J (1978) A study of the need for fibre by the growing New Zealand White rabbits. J Sci Food Agric 29, 640-648

Van Soest PJ, McQueen RW (1973) The chemistry and estimation of fibre. Proc Nutr Soc 32, 123-130 